\title{
Disease burden and related medical costs of rotavirus infections in
}

\section{Taiwan}

\author{
Chun-Yi Lu${ }^{1}$, Tsai-Ling Lauderdale ${ }^{2}$, Yin-Hua Fang³, Chung-Yi Wang1, Yu- \\ Huai $\mathrm{Ho}^{4}$, Che-Lun Hung${ }^{2}$, Luan-Yin Chang ${ }^{1}$, Chin-Yun Lee ${ }^{1}$ and Li- \\ Min Huang*1
}

\begin{abstract}
Address: ${ }^{1}$ Department of Pediatrics, National Taiwan University Hospital, Taipei, Taiwan, ${ }^{2}$ Division of Clinical Research, National Health Research Institutes, Zhunan, Taiwan, ${ }^{3}$ Department of Pediatrics, Min-Shen Hospital, Tao-Yun, Taiwan and ${ }^{4}$ Department of Pediatrics, Buddhist Tzu-Chi General Hospital, Hua-Lien, Taiwan

Email: Chun-Yi Lu - chunyi@ha.mc.ntu.edu.tw; Tsai-Ling Lauderdale - lauderdale@nhri.org.tw; Yin-Hua Fang - yinna@ha.mc.ntu.edu.tw; Chung-Yi Wang - tucle91@hotmail.com; Yu-Huai Ho - hoyuhuai@yahoo.com.tw; Che-Lun Hung - allen0104.tw@yahoo.com.tw; LuanYin Chang - ly7077@tpts6.seed.net.tw; Chin-Yun Lee - mfsun@ha.mc.ntu.edu.tw; Li-Min Huang* - lmhuang@ha.mc.ntu.edu.tw

* Corresponding author
\end{abstract}

Published: 15 December 2006

BMC Infectious Diseases 2006, 6:176 doi:10.1186/147/-2334-6-176
Received: 29 April 2006

Accepted: 15 December 2006

This article is available from: http://www.biomedcentral.com//47I-2334/6/I76

(C) 2006 Lu et al; licensee BioMed Central Ltd.

This is an Open Access article distributed under the terms of the Creative Commons Attribution License (http://creativecommons.org/licenses/by/2.0), which permits unrestricted use, distribution, and reproduction in any medium, provided the original work is properly cited.

\begin{abstract}
Background: The disease burden and associated medical costs of rotavirus infections in inpatient and outpatient sectors in Taiwan were examined in anticipation of the availability of new rotavirus vaccines.

Methods: The yearly national case number and medical costs for all for inpatients and outpatients with acute gastroenteritis (AGE) were extracted from the Bureau of National Health Insurance database in Taiwan according to ICD-9-CM codes. A retrospective study was also performed using records of children with AGE seen at three hospitals in Taiwan in 2001 to identify laboratory confirmed rotavirus infection cases. The annual incidence and related medical costs of AGE due to rotavirus infection were then estimated.

Results: Children $<5$ years old comprised $83.6 \%$ of inpatient and $62.0 \%$ of outpatient pediatric AGE cases in Taiwan in 200I. Rotavirus was the most common agent detected among AGE patients in this age group in the three hospitals, and was detected in 32.9\% (22I/672) of inpatient and 24\% (23/96) of outpatient stool specimens tested for microbial etiologies. An estimated 277,400 to 624,892 cases of rotavirus infections sought medical care in Taiwan in 200I, equaling one in 2 to 5 children $<5$ years old required medical care due to rotavirus infection. The incidence of hospitalization due to rotavirus infections was I,528-I,997/I00,000 for children $<5$ years old. The total associated medical costs due to rotavirus infection were estimated at US \$10-16 millions in Taiwan in 200I. Although the per-capita medical cost of rotavirus infection was lower in Taiwan than in the United States or Hong Kong, the personal economic burden was similar among the three places when normalized for gross national incomes per capita.
\end{abstract}

Conclusion: Infections caused by rotavirus constitute an important human and economic burden among young children in Taiwan. A safe and effective vaccine is urgently needed. 


\section{Background}

Rotavirus is the leading cause of severe childhood diarrhoea in developed and developing countries worldwide. Virtually all children could be infected before their fifth birthdays. It is estimated that each year rotavirus infections are responsible for approximately 2 million hospitalizations in children $<5$ years old worldwide $[1,2]$. The World Health Organization estimated that in 2002, over 400,000 deaths due to rotavirus in children $<5$ years old were preventable by vaccination [3]. Thus universal childhood immunization with rotavirus vaccine is expected to substantially reduce the mortality and morbidity due to rotavirus infections in immunocompetent children $[4,5]$.

Rotavirus infections have been reported to occur yearround in Taiwan [6,7]. Recently, in the first report from the Asian Rotavirus Surveillance Network (ARSN) by Bresee et al, rotavirus infection accounted for $28 \%$ to $59 \%$ (mean $45 \%$ ) of children $<5$ years old admitted to hospitals due to acute diarrhoea in Asia [8]. In a concurrent study of ARSN, rotavirus was detected in $43 \%$ of stool samples tested for etiology in children $<5$ years old admitted to 4 sentinel hospitals in Taiwan [9]. However, the national incidence of rotavirus disease in Taiwan is unknown, especially in the outpatient sector.

Taiwan implemented a national health insurance program in 1995, and by December 2000, over $96 \%$ of its $>22$-million population was covered under this universal healthcare system [10]. The Bureau of National Health Insurance (BNHI) in Taiwan maintains an extensive database on all inpatient and outpatient visits, including their medical diagnoses and medical expenditure. In anticipation of the availability of new rotavirus vaccines, we performed a retrospective study using available data from three hospitals and the BNHI database to estimate the burden of rotavirus disease and its associated medical costs to determine the potential economic impact with the implementation of a national vaccination program in Taiwan.

\section{Methods \\ Hospital data}

Three hospitals in Taiwan participated in this study. The National Taiwan University Hospital (NTUH) is a large 2,000-bed medical center located in the highly urbanized city of Taipei. The Min-Shen Hospital (MSH) is a regional hospital with 600 beds located in Tao-Yuan City. This is a satellite city of Taipei and includes more suburban areas. Both of these hospitals are located in the most densely populated northern regions of Taiwan. The Buddhist TzuChi General Hospital (TCH) is a 900-bed hospital in HuaLien City, which is located in the mountainous area of eastern Taiwan and serves a large rural area. This study was done independently of the ARSN studies.
We extracted medical and administrative records of all pediatric patients $<5$ years old with the principle diagnosis of acute gastroenteritis (AGE) who were seen in the outpatient department (including emergency rooms), and those admitted to these three hospitals between March 2001 and February 2002. While estimating the percentage of AGE attributable to rotavirus infections, patients whose records appeared both in the outpatients and inpatient sectors were counted as a single case in the inpatient sector. Only those whose stool samples were tested for both rotavirus and bacterial agents were taken into account. The causative microbes for AGE from patients who had stool specimens tested for microbial etiology were analyzed.

\section{Laboratory studies}

Stool samples submitted for microbial etiology testing were tested for rotaviral antigen by monoclonal antibody incorporated enzyme-linked immunosorbent assay according to the manufacturer's instructions (Rotaclone, Meridian Diagnostic, Cincinnati, OH, USA). Bacterial cultures were performed using MacConkey, xylose-lysinedesoxycholate, Hektoen enteric and thiosulfate-citratebile salts-sucrose agar, and Campylobacter Agar plates (BBL, Becton Dickinson Microbiology System, Cockeysville, MD, USA) following standard clinical microbiology protocols.

\section{Data from the Bureau of National Health Insurance (BNHI) database}

All the identification numbers of individuals were scrambled at BNHI before the database was made available for research purposes through a contract with the National Health Research Institutes. Because the database inclusive of all patient visits is tremendously large, we used the sampling database to perform the queries. The sampling database is a systematic sampling of every 500th outpatient visit and every 20th inpatient visit extracted from the allpatient records. For 2001, there were over 620,000 outpatient and over 140,000 inpatient records in the sampling database.

The BNHI database contains registration files, original claim information and reimbursement data. Medical diagnoses are coded according to the International Classification of Diseases, 9th revision, Clinical Modification (ICD-9-CM). We queried for patients 16 years old and younger with the following ICD-9-CM diarrhoea-associated diagnoses codes to analyze the number of AGE cases. The reimbursements associated with each visit and admission for these patients were extracted and analyzed. The codes include: 001 (cholera), 002 (typhoid and paratyphoid fever), 003 (salmonellosis), 004 (shigellosis), 005 (other bacterial food poisoning), 006 (amoebiasis), 007 (other protozoan intestinal diseases), 008 (intestinal 
infections due to other organisms including 00861 enteritis due to rotavirus), 009 (ill-defined intestinal infections), 558.9 (other and unspecified noninfectious gastroenteritis and colitis), and 787.91 (diarrhoea). Cases with the following codes were excluded in the current study: 003.2 (localized salmonella infections), 006.2 (amoebic liver abscess), 006.3 (hepatic amoebiasis), 006.4 (amoebic lung abscess), 006.5 (amoebic abscess of lung and liver), and 006.6 (amoebic skin ulceration).

\section{Estimation of Rotavirus Disease Burden and Medical Costs} Because the majority of rotavirus infections occurs in children $<5$ years old, surveillance of rotavirus infection usually defines a case of rotavirus diarrhoea as watery diarrhoea in a child $<5$ years old whose stool sample tested positive for rotavirus $[11,12]$. We adopted this rule and only cases of AGE in children $<5$ years old were taken into account in estimating disease burden or medical cost of rotavirus infections. The total number of rotavirus AGE cases was estimated by multiplying the total number of AGE cases in $<5$ years old children from the BNHI database and the percentages of laboratory confirmed rotavirus infections. Results from this study as well as those from the literature were used for this purpose.

Medical care expenses are reimbursed by the BNHI in Taiwan. The co-payment data (10\% co-payments for hospitalized patients and US \$1.5-3.0 for outpatients) are listed in the BNHI database along with reimbursement data and were included in our medical cost estimation. Nationwide total medical costs attributable to rotavirus infections in 2001 were estimated by multiplying the average BNHI reimbursements per AGE patient with the esti- mated annual case numbers of rotavirus infections. The monetary values in New Taiwan dollars (NT \$) were converted into United States dollars (US \$) based on the average exchange rate in 2001 ( 1 US $\$=33.8$ NT $\$$ ). The study was approved by the Clinical Research Ethics Committee of the National Taiwan University Hospital.

\section{Results}

\section{National Health Insurance System Data}

The nationwide case numbers of acute gastroenteritis (AGE) derived from the BNHI database is shown in Table 1 . There were a total of $2,829,740$ pediatric patient visits due to AGE, including 2,750,500 (97.2\%) in the ambulatory and $79,240(2.8 \%)$ in the inpatient sector in Taiwan in 2001. The majority of AGE occurred in children $<5$ years old, with this age group accounting for $62.0 \%$ $(1,704,000 / 2,750,500)$ and $83.6 \%(66,260 / 79,240)$ of pediatric outpatients and inpatients with AGE, respectively. However, most of the AGE cases were not coded for a specific etiologic agent. Instead, the most common codes reported were "Other and unspecified noninfectious gastroenteritis and colitis" (ICD-9-CM 558.9) and "Ill-defined intestinal infections" (ICD-9-CM 009). These two codes together accounted for $95.3 \%$ $[(836,500+1,783,500) / 2,750,500]$ of outpatients and $82.0 \%[(9,500+55,460) / 79,240]$ of inpatients with AGE related diagnoses.

Enteritis due to rotavirus (ICD-9-CM 00861) was not reported in any outpatients and was reported only in a small number $(0.4 \%, 320 / 79,240)$ of inpatients. All of the rotavirus AGE inpatients were in children $<5$ years old. Therefore, the prevalence of rotavirus AGE could not be

Table I: Case numbers of acute gastroenteritis with various ICD-9-CM codes in children in Taiwan, 200 Ia.

\begin{tabular}{|c|c|c|c|c|c|c|c|c|c|c|c|c|c|}
\hline \multirow[t]{2}{*}{ Sectors } & \multirow[t]{2}{*}{ Age (years old) } & \multicolumn{11}{|c|}{ Case numbers of various acute gastroenteritis associated ICD-9-CMb } & \multirow[t]{2}{*}{$\% d$} \\
\hline & & 001 & 002 & 003 & 004 & 005 & 007 & $008^{c}$ & 009 & 558.9 & 787.91 & Total & \\
\hline \multirow[t]{3}{*}{ Outpatient } & $<5$ & 500 & 1,000 & 40,000 & 500 & 1,000 & 500 & 34,500 & 540,000 & $1,085,500$ & 500 & $1,704,000$ & 62.0 \\
\hline & $5-16$ & 0 & 0 & 20,500 & 0 & 5,000 & 0 & 24,000 & 296,500 & 698,000 & 2,500 & $1,046,500$ & 38.0 \\
\hline & $0-16$ & 500 & 1,000 & 60,500 & 500 & 6,000 & 500 & 58,500 & 836,500 & $\mathrm{I}, 783,500$ & 3,000 & $2,750,500$ & \\
\hline \multirow[t]{3}{*}{ Inpatient } & $<5$ & 0 & 20 & 4,320 & 100 & 40 & 0 & 8,180 & 8,220 & 45360 & 20 & 66,260 & 83.6 \\
\hline & $5-16$ & 0 & 0 & 440 & 200 & 80 & 0 & 880 & 1280 & 10100 & 0 & 12,980 & 16.4 \\
\hline & $0-16$ & 0 & 20 & 4,760 & 300 & 120 & 0 & 9060 & 9500 & 55460 & 20 & 79,240 & \\
\hline \multirow[t]{3}{*}{ Total } & $<5$ & 500 & 1,020 & 44,320 & 600 & 1040 & 500 & 42680 & 548220 & $1,130,860$ & 520 & $1,770,260$ & 62.6 \\
\hline & $5-16$ & 0 & 0 & 20,940 & 200 & 5080 & 0 & 24880 & 297,780 & 708100 & 2500 & $1,059,480$ & 37.4 \\
\hline & $0-16$ & 500 & 1,020 & 65,260 & 800 & 6,120 & 500 & 67,560 & 846,000 & $1,838,960$ & 3,020 & $2,829,740$ & \\
\hline
\end{tabular}

\footnotetext{
a Extrapolated from the Bureau of National Health Insurance 200I sampling database in Taiwan (see Methods for details).

b ICD-9-CM: International Classification of Diseases, 9th revision, Clinical Modification. 00I, cholera; 002, typhoid and paratyphoid fever; 003, salmonellosis [excluding 003.2 (localized Salmonella infections)]; 004, shigellosis; 005, other food poisoning (bacterial); 007, other protozoan intestinal diseases; 008, intestinal infections caused by other organisms; 009, ill-defined intestinal infections; 558.9, other and unspecified noninfectious gastroenteritis and colitis; 787.91, diarrhoea. There were no cases of 006 (amoebiasis) reported.

c Including 320 cases of ICD-9-CM 008.6I, rotavirus.

dPercentage of all 0-16 years old cases.
} 
obtained directly from the BNHI database. Because the majority of pediatric AGE cases occurred in $<5$ years old children, and because enteritis due to rotavirus was reported only in this age group in the BNHI database, our subsequent analysis on rotavirus disease burden focused on this age group.

\section{Hospital data}

During the one-year study period, 5,015 children $<5$ years old with AGE were treated at the three participating hospitals. Of these 5,015 patients, $24.3 \%(1,219)$ were admitted due to severe diarrhoea and/or vomiting with dehydration, and were hospitalized for 3 to 5 days (mean $=4$ days). Although laboratory diagnosis for etiology was performed on only a small proportion of the outpatients $(2.5 \%, 96 / 3,796)$, over half of the hospitalized patients $(55.1 \%, 672 / 1,219)$ were tested for an etiologic agent including both rotavirus and bacteria (Table 2). In AGE inpatients, rotavirus accounted for $>33 \%$ in the 2 hospitals in the north. Although it was detected in lower proportion $(18.3 \%)$ in the hospital in the east (TCH), it was still the most common agent detected in that hospital. Overall, rotavirus was the leading cause of AGE in these three hospitals, accounting for $24.0 \%$ (23/96) of outpatients and $32.9 \%(221 / 672)$ of inpatients tested.

\section{Direct medical costs for AGE}

The full medical costs, including BNHI reimbursements and co-payments for children $<5$ years old with AGE-associated ICD9 codes based on the BNHI data are shown in Table 3. The BNHI reimbursements for AGE typically include hospital room charge and nursing fee (40.7\%), pharmacy and treatment-related charges $(29.3 \%)$, laboratory services $(14.6 \%)$, and physician fee $(15.4 \%)$ (data not shown). The values in Table 3 are full medical costs including BNHI reimbursements, co-payments, and all governmental subsidies for certain subpopulations. On average, it cost US $\$ 10.8$ for each ambulatory patient visit and US $\$ 336.0$ for each hospitalization due to AGE. Although children $<5$ years old requiring hospitalization comprised approximately 3.7\% $(66,260 / 1,770,260)$ of children in this age group with AGE (Table 1), over half $(54.8 \%, 22,265,691 / 40,600,883)$ of the medical expenses were accrued by these hospitalized patients (Table 3 ).

\section{Case numbers of rotavirus $A G E$ in children $<\mathbf{5}$ years old}

We used a range of $32.9-43 \%$ to estimate the total number of cases of children $<5$ years old hospitalized with rotavirus AGE. These numbers were based on results from our study of three hospitals, which showed that $32.9 \%$ of $<5$ years old children hospitalized due to AGE were attributable to rotavirus infection, as well as the study of Chen et al [9], which found $43 \%$ of the same age group of AGE patients admitted to 4 sentinel hospitals in Taiwan were due to rotavirus infection. For outpatients, we used a range of $15-35 \%$ based on the report of Glass et al [13] since the $24.0 \%$ of our three hospital data fall within this range.

The annual case number for rotavirus AGE would therefore be $255,600-596,400(1,704,000 \times 15-35 \%)$ for outpatients and 21,800-28,492 (66,260 × 32.9-43\%) for inpatients, for a total of 277,400-624,892 episodes of rotavirus infections in children $<5$ years old needing medical care in 2001 in Taiwan (Table 4). Since the population of children $<5$ years old was $1,426,759$ in Taiwan in 2001 [14], the incidence of rotavirus infection in children this age group would be $19.4-43.8 \%(277,400-624,892 /$ $1,426,759)$. In other words, an estimated 1 out of every 2 to 5 children $<5$ years old in Taiwan could have rotavirus

Table 2: Number of cases and distribution of etiological diagnoses in children $<5$ years old with acute gastroenteritis (AGE) seen at three hospitals in Taiwan in $200 \mathrm{I}^{\mathrm{a}}$.

\begin{tabular}{|c|c|c|c|c|}
\hline Cases of AGE in: & NTUH & $\mathrm{MSH}$ & $\mathrm{TCH}$ & Total of 3 hospitals \\
\hline Outpatients & I,274 & 1,679 & 843 & 3,796 \\
\hline Tested for etiology, $\mathrm{N}^{\mathrm{b}}$ & 67 & 12 & 17 & 96 \\
\hline Positive for rotavirus, $\mathrm{N}(\%)^{\mathrm{c}}$ & $18(26.9)$ & $2(16.7)$ & $3(17.6)$ & $23(24.0)$ \\
\hline Positive for Salmonella, N (\%) & $9(13.4)$ & $2(16.7)$ & $4(23.5)$ & $15(15.6)$ \\
\hline Positive for Shigella, N (\%) & 0 & 0 & 0 & 0 \\
\hline Positive for Campylobacter, N (\%) & $\mathrm{I}(\mathrm{I} .5)$ & $N T^{d}$ & NT & - \\
\hline Inpatients & 408 & 378 & 433 & 1,219 \\
\hline Tested for etiology, $\mathrm{N}$ & 287 & 292 & 93 & 672 \\
\hline Positive for rotavirus, $\mathrm{N}(\%)$ & $106(36.9)$ & $98(33.6)$ & $17(18.3)$ & $221(32.9)$ \\
\hline Positive for Salmonella, N (\%) & $34(11.8)$ & $26(8.9)$ & $8(8.6)$ & $68(10.1)$ \\
\hline Positive for Shigella, N (\%) & 0 & $2(0.7)$ & $12(12.9)$ & $14(2.1)$ \\
\hline Positive for Campylobacter, N (\%) & $\mathrm{I}(0.3)$ & NT & NT & - \\
\hline
\end{tabular}

a NTUH, National Taiwan University Hospital; MSH, Min-Shen Hospital; TCH, Buddhist Tzu-Chi General Hospital.

b Number of AGE cases tested for both rotavirus and bacterial agents.

c Percentage of those tested.

dNT, Not tested. 
Table 3: National health insurance reimbursements and co-pays for children $<5$ years old with various acute gastroenteritis ICD-9-CM codes in Taiwan in 2001 .

\begin{tabular}{|c|c|c|c|c|c|c|c|c|c|c|c|c|}
\hline \multirow[t]{2}{*}{ Sectors } & \multicolumn{12}{|c|}{ Medical costs (in US \$) for various acute gastroenteritis associated ICD-9-CMa } \\
\hline & 001 & 002 & 003 & 004 & 005 & 007 & 008 & 009 & 558.9 & 787.91 & Total & Average ${ }^{b}$ \\
\hline Outpatient & 5,237 & 8,905 & 394,497 & 7,012 & 10,828 & 5,636 & 350,178 & $5,706,479$ & $11,900,784$ & 5,636 & $18,395,192$ & 10.8 \\
\hline Inpatient & 0 & 3,353 & $1,986,493$ & 30,337 & 8,383 & 0 & $2,800,989$ & $2,4 \mid 3,857$ & $15,018,609$ & 3,668 & $22,265,691$ & 336.0 \\
\hline Total & 5,237 & 12,259 & $2,380,991$ & 37,349 & 19,211 & 5,636 & $3,151,167$ & $8,120,336$ & $26,919,393$ & 9,304 & $40,660,883$ & - \\
\hline
\end{tabular}

a ICD-9-CM: International Classification of Diseases, 9th revision, Clinical Modification. 002, typhoid and paratyphoid fever; 003, salmonellosis [excluding 003.2 (localized Salmonella infections)]; 004, shigellosis; 005, other food poisoning (bacterial); 007, other protozoan intestinal diseases; 008, intestinal infections caused by other organisms; 0086, enteritis due to specified virus; 0086I, rotavirus; 009, ill-defined intestinal infections; 558.9, other and unspecified noninfectious gastroenteritis and colitis; 787.9I, diarrhoea. There were no cases with the ICD-9-CM of 006 (amoebiasis) in this age group.

b Total medical costs divided by total case numbers (I,704,000 for outpatients and 66,260 for inpatients) (See Table I for details).

infection that needed medical care, and the estimated hospitalization rate for rotavirus infections would be $1,528-$ $1,997 / 100,000(21,800-28,492 / 1,426759)$ in children of this age group.

The estimated medical expenses due to rotavirus infections in children $<5$ years old were extrapolated using the average cost per patient multiplied by the number of estimated rotavirus cases (Table 4). Based on these calculations, an estimated total medical expenditure of US \$10.1-16.0 million, including US \$2.8-6.4 million for outpatients and US \$7.3-9.6 million for inpatients was spent in 2001 for treating rotavirus infections in young children in Taiwan.

\section{Comparison of medical cost for rotavirus hospitalization in Taiwan, Hong Kong, and USA}

We also compared the annual direct medical costs for hospitalizations due to rotavirus infections in Taiwan (US $\$ 7-10$ million) with data available from USA (US \$217 million in 1996 dollar) [15] and Hong Kong (US \$4 million in 2001-2003) for children $<5$ years old [16]. After normalization with population and gross national income (GNI) per capita for $2001[14,17]$, the economic burden could be compared among different countries. Although the direct medical cost for rotavirus infections per capita was lower in Taiwan (US \$0.31-0.44) than in Hong Kong (US \$0.59) or USA (US \$0.76), the personal economic burden indexes were similar among Taiwan (1.92-2.72), Hong Kong (2.28), and USA (2.19) when normalized for GNI per capita (Table 5).

\section{Discussion}

This is the first analysis of the prevalence and medical costs of childhood rotavirus AGE for both inpatients and outpatients in Taiwan. This analysis confirmed rotavirus as a leading cause of AGE in children $<5$ years old who sought medical care in Taiwan. Nearly one third (32.9\%) of hospitalized AGE cases were caused by rotavirus. This finding is comparable to data reported from previous studies in other countries, which ranged from $25 \%$ to $50 \%$. Rotavirus accounted for $30-50 \%$ of diarrhoeal hospitalization in $<5$ years old children in England [18], 27\% in diarrhoeal consultations in El Salvador [19], and $42 \%$ of diarrhoeal admission in Argentina [20]. In New York, rotavirus infection was reported in $>30 \%$ of diarrhoea cases in children younger than 5 years of age [21].

In a separate study of 4 hospitals in Taiwan, Chen et al found that $43 \%$ of AGE cases in $<5$ years old children hospitalized were attributable to rotavirus [9]. There are several explanations for the difference in our data $(32.9 \%)$ and that of Chen et al. In two of the hospitals we studied, over $33 \%$ of the young children with AGE hospitalized were attributable to rotavirus. The lower proportion $(18 \%)$ of the patients with rotavirus AGE at the third hos-

Table 4: The direct medical cost for rotavirus acute gastroenteritis (AGE) in <5 years old children in Taiwan, 200 I

\begin{tabular}{|c|c|c|c|c|c|}
\hline \multirow[t]{2}{*}{ Sector } & \multirow[t]{2}{*}{ Medical cost (in US \$) per case } & \multicolumn{2}{|c|}{ Total AGE } & \multicolumn{2}{|c|}{ Estimated rotavirus AGE } \\
\hline & & Case no. & Total medical cost & Case no. & Total medical cost \\
\hline Outpatient & 10.8 & $\mathrm{I}, 704,000$ & $18,395,192$ & $255,600-596,400^{a}$ & $2,760,480-6,441,120$ \\
\hline Inpatient & 336.0 & 66,260 & $22,265,691$ & $21,800-28,492^{b}$ & $7,324,800-9,573,312$ \\
\hline Total & - & $1,770,260$ & $40,660,883$ & $277,400-624,892$ & $10,085,280-16,014,432$ \\
\hline
\end{tabular}

a The range of $15-35 \%$ was used for estimating outpatient rotavirus AGE case number based on Glass et al., (Reference [13]).

b For estimation of hospitalized rotavirus AGE case number, $32.9 \%$ was used as the lower end based on the three hospital data in the present study and $43 \%$ was used as the upper end based on study of Chen et al (Reference [9]). 
Table 5: Normalized medical costs for rotavirus acute gastroenteritis (AGE) hospitalizations in Taiwan, Hong Kong, and the United States (USA)

\begin{tabular}{|c|c|c|c|}
\hline & Taiwan & Hong Kongb & USAc \\
\hline Direct medical cost for rotavirus AGE (in US \$) & $7-10 \mathrm{mil}$ & $4 \mathrm{mil}$ & $217 \mathrm{mil}$ \\
\hline Population ${ }^{\mathrm{a}}$ & $22.9 \mathrm{mil}$ & $6.8 \mathrm{mil}$ & $285.3 \mathrm{mil}$ \\
\hline Medical cost per capita $(A)$ & $0.31-0.44$ & 0.59 & 0.76 \\
\hline Gross National Incomes (GNI) per capita ${ }^{a}(B)$ & 16,170 & 26,060 & 34,800 \\
\hline Normalized economic burden index due to rotavirus AGE $\left(A / B \times 10^{5}\right)$ & $1.92-2.72$ & 2.28 & 2.19 \\
\hline
\end{tabular}

a Population and GNI per capita in 200 I was used [References I4 and I7].

b The Hong Kong study was based on hospital data of 200I-2003 [reference I6].

c Because the direct medical costs estimate for USA was reported in 1996 dollars [reference 15], and we used the population and GNI data from

200I, the upper range of their sensitivity for cost estimate was used (4,346 US $\$$ per hospitalization $\times 50,000$ hospitalizations)

pital is likely due to the fact that eastern region of Taiwan where the hospital is located is the least populated mountainous area and one where bacterial dysentery remains an important infectious disease. Most other parts of Taiwan, including where the other two hospitals we studies are located, are highly urbanized and more densely populated. The lower rate of rotavirus infections in the hospital in the east might have underrepresented rotavirus in that region thus lowering our estimation for the whole country. Another possible explanation is that not all children with AGE in the three hospitals we studied had stool specimens tested for microbial etiology. Different testing practices might have led to an underestimation of the incidence of rotavirus infection. We therefore used the $43 \%$ reported by Chen et al [9] as the upper range and our $32.9 \%$ as the lower range to estimate the rotavirus disease burden in children hospitalized due to AGE in Taiwan.

The true incidence of rotavirus infections in the outpatient sectors is unknown because pediatricians tend not to perform rotavirus testing in typical cases of mild watery diarrhoea. This is especially true for the outpatient sector in Taiwan as evidenced by the fact that less than $3 \%$ of the outpatients with AGE were tested for etiology in the three hospitals we studied. We feel that the use of 15-35\% range reported by Glass et al [13] provides a good estimate for outpatient rotavirus disease burden in Taiwan since rotavirus was detected in $24 \%$ of the outpatients tested in these three hospitals.

Our results indicated that the disease burden of rotavirus infections could be higher in Taiwan than many other countries. An estimated one out of every 2 to 5 children younger than 5 years of age sought medical care due to rotavirus infections in Taiwan in 2001. The risks of children in the same age group requiring an outpatient visit due to rotavirus infection were estimated to be 1 out of 7 in the United States and 1 out of 5 globally [13]. Our estimation of the annual incidence of hospitalization due to rotavirus infections of $1,528-1,997 / 100,000$ in the $<5$ years old children in Taiwan is also higher than many countries, including Finland $(610 / 100,000$ in 19851995) [22], and Australia (750-870/100,000 in 19911996) [23][24]. We do not have any biological explanation for the high hospitalization rate due to rotavirus infections in Taiwan. An earlier report revealed the incidence of rotavirus disease is similar in children in different countries, regardless of the degree of development [1]. The high hospitalization rate in Taiwan might be due to the easy accessibility to and relatively low cost of health care. With the support of the National Health Insurance system, the medical costs are affordable to most of the population. Children with moderate to severe diarrhoea with early signs of dehydration are frequently admitted to the hospitals in Taiwan.

Of noteworthy is that although valuable information can be obtained from the extensive database maintained by the Bureau of National Health Insurance (BNHI) in Taiwan, very few AGE cases were coded as enteritis due to rotavirus (ICD9-00861) in the BNHI database. Although children with gastroenteritis who have no pathogen isolated should be correctly coded as ICD 009 (ill-defined intestinal infections), the BNHI data showed that ICD code 558.9 (other and unspecified noninfectious gastroenteritis and colitis) was often used instead. Earlier studies by Nelson et al on rotavirus disease burden also incorporated codes 558.9 and 787.91 (diarrhoea) into their analysis of disease burden of rotavirus infections [11]. There are several explanations for the non-specific ICD coding in Taiwan. Rotavirus AGE is clinically indistinguishable from AGE caused by other pathogens. Physicians in Taiwan also do not have the incentives to look for specific etiologic agents because insurance reimbursement is not higher for more specific diagnostic ICD coding. Stool testing is seldom done by practitioners or in local hospitals. Thus laboratory diagnosis and specific ICD coding should be encouraged to increase our understanding of the roles different etiologic agents play in causing diseases in Taiwan's patients. 
We also showed that although the per-capita medical cost of rotavirus infection was lower in Taiwan than that of the United States and Hong Kong, the personal economic burden was similar among the three places when normalized for gross national incomes per capita. Therefore, people in countries with different degrees of development have similar economic burdens from rotavirus infections. Other than the direct medical costs, one must also take into account associated indirect costs including an out of pocket registration fee for each outpatient visit (US \$1.5$3.0)$, extra diapers and laundry, special diets, transportation, lost of income from work, and additional child care needs resulting from children having rotavirus infections. Thus the social and economical burden of rotavirus infections could be in actuality much higher than the abovementioned figures.

\section{Conclusion}

Rotavirus remains a leading etiologic agent for acute gastroenteritis in children $<5$ years old in Taiwan. Even though mortality from rotavirus infection is no longer a significant problem due to easy access to medical care in Taiwan, the morbidity due to rotavirus is still very high. An estimated one out of every 2 to 5 children in the $<5$ years old group required medical care due to rotavirus infection in Taiwan in 2001 with an estimated annual medical expenditure of US $\$ 10-16$ million. It is hoped that preventive measures, including introduction of safe and effective rotavirus vaccines, would decrease the human and economic burdens of rotavirus disease in Taiwan.

\section{Competing interests}

LMH is currently a principal investigator and CYL is a coinvestigator of a phase 3 rotavirus vaccine study funded by GlaxoSmithKline. Both LMH and CYL have received lecture fees and travel support from Merck and GlaxoSmithKline. Both companies have licensed rotavirus vaccines. The other authors declare they have no competing interests.

\section{Authors' contributions}

CYLu was responsible for data collection and analysis, and writing of the manuscript. TLL was responsible for collection and analysis of the data from the Bureau of National Health Insurance (BNHI) database and participated in manuscript writing. CLH assisted in the BNHI data collection. YHF, CYW, YHH, LYC and CYLee participated in the collection and analysis of data from the three participating hospitals. LMH designed and supervised the execution of the study and manuscript writing. All authors have read and approved the final manuscript.

\section{Acknowledgements}

The authors are grateful to Dr. Calvin M. Kunin for his careful review of the manuscript.

\section{References}

I. Parashar UD, Hummelman EG, Bresee JS, Miller MA, Glass RI: Global illness and deaths caused by rotavirus disease in children. Emerg Infect Dis 2003, 9:565-572.

2. Miller MA, McCann L: Policy analysis of the use of hepatitis B, Haemophilus influenzae type b-, Streptococcus pneumoniae-conjugate and rotavirus vaccines in national immunization schedules. Health Econ 2000, 9:19-35.

3. World Health Organization Immunization surveillance, assessment and monitoring: Estimation of disease burden and cost effectiveness. [http://www.who.int/immunization monitoring/bur den].

4. Rotavirus vaccine for the prevention of rotavirus gastroenteritis among children. Recommendations of the Advisory Committee on Immunization Practices (ACIP). MMWR Recomm Rep 1999, 48: I-20.

5. de Quadros CA, Santos J: Rotavirus: the search for the next generation vaccine. Pediatr Infect Dis J 2004, 23:SI47-SI 48.

6. Tsai $\mathrm{CH}$, Chiu $\mathrm{HH}$, Abe $\mathrm{T}$ : Epidemiologic features of rotavirus infection in Taiwan: a review. Pediatr Int 2000, 42:4II-4I4.

7. Chiu TF, Lee CN, Lee PI, Kao CL, Lin HC, Lu CY, Tseng HY, Hsu HL, Lee CY, Huang LM: Rotavirus gastroenteritis in children: 5-year experience in a medical center. J Microbiol Immunol Infect 2000, 33: $181-186$.

8. Bresee J, Fang ZY, Wang B, Nelson EA, Tam J, Soenarto Y, Wilopo SA, Kilgore P, Kim JS, Kang JO, Lan WS, Gaik CL, Moe K, Chen KT, Jiraphongsa C, Ponguswanna Y, Nguyen VM, Phan VT, Le TL, Hummelman E, Gentsch JR, Glass R: First report from the Asian Rotavirus Surveillance Network. Emerg Infect Dis 2004, I 0:988-995.

9. Chen KT, Chen PY, Tang RB, Huang YF, Lee PI, Yang JY, Chen HY, Bresee J, Hummelman E, Glass R: Sentinel hospital surveillance for rotavirus diarrhea in Taiwan, 2001-2003. J Infect Dis 2005, I 92 Suppl I:S44-S48.

10. Bureau of National Health Insurance: National Health Insurance Annual Statistical Report [in Chinese] Taipei, Bureau of National Haelth Insurance, Taiwan ; 2003.

II. Nelson EA, Tam JS, Bresee JS, Poon KH, $\mathrm{Ng} \mathrm{CH}$, Ip KS, Mast TC, Chan PK, Parashar UD, Fok TF, Glass RI: Estimates of rotavirus disease burden in Hong Kong: hospital-based surveillance. J Infect Dis 2005, I 92 Suppl I:S7I-S79.

12. World Health Organisation: Generic protocol for (i) hospital based surveillance to estimate the burden of rotavirus gastroenteritis in children and (ii) a community-based survey on utilization of health care services for gastroenteritis in children. Field test version (WHO/V\&B/02.15). Geneva, WHO; 2002.

13. Glass RI, Parashar UD, Bresee JS, Turcios R, Fischer TK, Widdowson $M A$, Jiang $B$, Gentsch JR: Rotavirus vaccines: current prospects and future challenges. Lancet 2006, 368:323-332.

14. Department of Statistics, Ministry of Interior, ROC. Statistical Yearbook of Interior: Population by age. [http://www.moi.gov.tw/stat/ index.asp].

15. Tucker AW, Haddix AC, Bresee JS, Holman RC, Parashar UD, Glass $\mathrm{RI}$ : Cost-effectiveness analysis of a rotavirus immunization program for the United States. JAMA 1998, 279:137|-I376.

16. Nelson EA, Tam JS, Yu LM, Ng YC, Bresee JS, Poon KH, Ng CH, Ip KS, Mast TC, Chan PK, Parashar UD, Fok TF, Glass RI: Hospitalbased study of the economic burden associated with rotavirus diarrhea in Hong Kong. J Infect Dis 2005, 192 Suppl I:S64-S70.

17. The World Bank Group. WDI data query. [http://devdata.world bank.org/data-query/].

18. Ryan MJ, Ramsay M, Brown D, Gay NJ, Farrington CP, Wall PG: Hospital admissions attributable to rotavirus infection in England and Wales. J Infect Dis 1996, I74 SuppI I:SI2-SI8.

19. Guardado JA, Clara WAW, Turcios RM, Fuentes RA, Valencia D, Sandoval R, Barahona F, Bresee JS, Glass RI: Rotavirus in EI Salvador: an outbreak, surveillance and estimates of disease burden, 2000-2002. Pediatr Infect Dis J 2004, 23:SI56-SI 60.

20. Bok K, Castagnaro N, Borsa A, Nates S, Espul C, Fay O, Fabri A, Grinstein S, Miceli I, Matson DO, Gomez JA: Surveillance for rotavirus in Argentina. J Med Virol 2001, 65:190-198.

21. Chang HG, Glass RI, Smith PF, Cicirello HG, Holman RC, Morse DL: Disease burden and risk factors for hospitalizations associated with rotavirus infection among children in New York State, 1989 through 2000. Pediatr Infect Dis J 2003, 22:808-8I4. 
22. Vesikari T, Rautanen $\mathrm{T}$, Von Bonsdorff $\mathrm{CH}$ : Rotavirus gastroenteritis in Finland: burden of disease and epidemiological features. Acta Paediatr Suppl 1999, 88:24-30.

23. Ferson MJ: Hospitalisations for rotavirus gastroenteritis among children under five years of age in New South Wales. Med J Aust 1996, 164:273-276.

24. Holmes WR: Rotavirus infection and rates of hospitalisation for acute gastroenteritis in young children in Australia, 19931996. Med J Aust 1999, 170:189-190.

\section{Pre-publication history}

The pre-publication history for this paper can be accessed here:

http://www.biomedcentral.com/1471-2334/6/176/pre pub

Publish with Bio Med Central and every scientist can read your work free of charge

"BioMed Central will be the most significant development for disseminating the results of biomedical research in our lifetime. "

Sir Paul Nurse, Cancer Research UK

Your research papers will be:

- available free of charge to the entire biomedical community

- peer reviewed and published immediately upon acceptance

- cited in PubMed and archived on PubMed Central

- yours - you keep the copyright

Submit your manuscript here:

http://www.biomedcentral.com/info/publishing_adv.asp 